PROCEEDINGS OF THE

AMERICAN MATHEMATICAL SOCIETY

Volume 133, Number 4, Pages 1213-1221

S 0002-9939(04)07862-1

Article electronically published on November 19, 2004

\title{
GEOMETRIC INEQUALITIES FOR A CLASS OF EXPONENTIAL MEASURES
}

\author{
HERMANN KOENIG AND NICOLE TOMCZAK-JAEGERMANN
}

(Communicated by David R. Larson)

\begin{abstract}
Using $M$-ellipsoids we prove versions of the inverse Santaló inequality and the inverse Brunn-Minkowski inequality for a general class of measures replacing the usual volume on $\mathbb{R}^{n}$. This class contains in particular the Gaussian measure on $\mathbb{R}^{n}$.
\end{abstract}

In this note we consider versions of some geometric inequalities (of an isomorphictype) for a natural class of exponential log-concave measures, replacing the usual volume in $\mathbb{R}^{n}$. These are versions of Milman's inverse Brunn-Minkowski inequality ([M]) and Bourgain-Milman's inverse Santaló inequality ([BM]$)$, which have played an important role in the convex geometric analysis and the asymptotic theory of normed spaces during the last fifteen years (cf. also $[\mathrm{P}$ ). Both these inequalities can be viewed as a consequence of the existence, for any symmetric convex body in $\mathbb{R}^{n}$, of a special ellipsoid, called nowadays an $M$-ellipsoid ([M]$)$, which, in a sense, reflects volumetric properties of the body. In this note we show that the same ellipsoid also reflects, in an analogous way, properties of the body with respect to a large class of exponential (log-concave) measures on $\mathbb{R}^{n}$. This class contains in particular the Gaussian measure on $\mathbb{R}^{n}$.

The Brunn-Minkowski inequality and other geometric inequalities have been developed and extended over the years, and we refer the reader e.g. to [G] for a recent survey on this subject. We also mention papers [C] and especially [CFM], which partially motivate the present note, and where, among other results, connections between complex interpolation and the Brunn-Minkowski and Santaló inequalities have been developed.

Let us recall basic notation. We consider $\mathbb{R}^{n}$ with the standard Euclidean structure and the Euclidean unit ball denoted by $B_{2}^{n}$. The canonical Euclidean norm on $\mathbb{R}^{n}$ is denoted by $|\cdot|$, and the corresponding inner product by $\langle\cdot, \cdot\rangle$. An ellipsoid $D \subset \mathbb{R}^{n}$ is a linear image $D=u\left(B_{2}^{n}\right)$ for an invertible operator $u$ on $\mathbb{R}^{n}$.

By a symmetric convex body we mean a centrally symmetric convex compact set with a non-empty interior. For a symmetric convex body $K$ in $\mathbb{R}^{n}$ the polar body $K^{0}$ is defined by

$$
K^{0}:=\left\{x \in \mathbb{R}^{n}||\langle x, y\rangle \mid \leq 1 \text { for every } y \in K\right\} .
$$

Received by the editors December 21, 2003.

2000 Mathematics Subject Classification. Primary 46B20, 52A21.

Key words and phrases. Asymptotic geometric analysis, Santaló and Brunn-Minkowski inequalities, $M$-ellipsoids.

The second named author holds the Canada Research Chair in Geometric Analysis.

(C)2004 American Mathematical Society Reverts to public domain 28 years from publication 
The $n$-dimensional volume of a set $K$ in $\mathbb{R}^{n}$ is denoted by $|K|$. For two sets $K, L \subset \mathbb{R}^{n}$, we denote the Minkowski sum by $K+L$, i.e., the set of all $x+y$ where $x \in K$ and $y \in L$.

We shall consider a number of properties of measures $\mu$ on $\mathbb{R}^{n}$ :

(i) there exists $C_{1}$ such that for every symmetric convex set $B \subset \mathbb{R}^{n}$ and every $x \in \mathbb{R}^{n}$ we have $(\mu(x+B))^{1 / n} \leq C_{1}(\mu(B))^{1 / n}$;

(ii) there exists $C_{2}$ such that for every symmetric convex body $K \subset \mathbb{R}^{n}$ and every $\lambda \geq 1$ we have $(\mu(\lambda K))^{1 / n} \leq C_{2} \lambda(\mu(K))^{1 / n}$;

(iii) for some interval $\left[a_{1}, a_{2}\right] \subset[0, \infty)$ and some $C_{3}$, the following inequality holds, for every $t \in(0,1)$ and every $\lambda_{1}, \lambda_{2} \in\left[a_{1}, a_{2}\right]$,

$$
\mu\left(\left(t \lambda_{1}+(1-t) \lambda_{2}\right) B_{2}^{n}\right)^{1 / n} \leq C_{3}\left(t \mu\left(\lambda_{1} B_{2}^{n}\right)^{1 / n}+(1-t) \mu\left(\lambda_{2} B_{2}^{n}\right)^{1 / n}\right) .
$$

Let us now recall the notion of an $M$-ellipsoid which is a starting point for our investigations. It requires a standard notation. If $K$ and $L$ are two sets on $\mathbb{R}^{n}$, by $N(K, L)$ we denote the covering number of $K$ by $L$, i.e., the minimal number of translations of $L$ needed to cover $K$.

Definition 1. Let $K \subset \mathbb{R}^{n}$ be a symmetric convex body. An ellipsoid $D \subset \mathbb{R}^{n}$ is an $M$-ellipsoid for $K$ with constant $C$ if the covering numbers satisfy

$$
\max \left\{N(K, D), N(D, K), N\left(K^{0}, D^{0}\right), N\left(D^{0}, K^{0}\right)\right\} \leq \exp (C n) .
$$

This notion was introduced by Milman in [M] who proved that there is an absolute constant $C_{0}>0$ such that for every symmetric convex body $K$ in $\mathbb{R}^{n}$ there exists an $M$-ellipsoid for $K$ with constant $C_{0}$. Throughout this paper we shall use the notation $C_{0}$ for such a constant in (1). Another proof of the existence of an $M$-ellipsoid can be found, e.g., in $[\mathrm{P}]$.

Our first result shows that, for a natural class of measures, any $M$-ellipsoid satisfies estimates analogous to (7.2) in $[\mathrm{P}]$.

Theorem 2. Let $K \subset \mathbb{R}^{n}$ be a symmetric convex body and let $D \subset \mathbb{R}^{n}$ be an $M$-ellipsoid for $K$ with constant $C_{0}$. Assume that $\mu$ is a measure on $\mathbb{R}^{n}$ satisfying conditions (i) and (ii). Then

$$
\left(\frac{\mu(K+D)}{\mu(K \cap D)} \frac{\mu\left(K^{0}+D^{0}\right)}{\mu\left(K^{0} \cap D^{0}\right)}\right)^{1 / n} \leq C^{\prime}
$$

where $C^{\prime}=\left(2 C_{1} C_{2} \exp \left(C_{0}\right)\right)^{4}$.

Proof. Since $N(K+D, 2 D) \leq N(K, D) \leq \exp \left(C_{0} n\right)$, then using properties (i) and (ii) we get $\mu(K+D) \leq N(K+D, 2 D) C_{1}^{n} \mu(2 D) \leq \exp \left(C_{0} n\right) C_{1}^{n} C_{2}^{n} 2^{n} \mu(D)$.

On the other hand, since $N(D, 2(K \cap D)) \leq N(D, K) \leq \exp \left(C_{0} n\right)$, then using (i) and (ii) again we get

$$
\mu(D) \leq N(D, 2(K \cap D)) C_{1}^{n} \mu(2(K \cap D)) \leq \exp \left(C_{0} n\right) C_{1}^{n} C_{2}^{n} 2^{n} \mu(K \cap D) .
$$

Putting these estimates together we get

$$
\left(\frac{\mu(K+D)}{\mu(K \cap D)}\right)^{1 / n} \leq 4 C_{1}^{2} C_{2}^{2} \exp \left(2 C_{0}\right)
$$

The estimate for polars follows the same way. 
If $K, B \subset \mathbb{R}^{n}$ are two symmetric convex bodies with $M$-ellipsoids $D, D_{1} \subset \mathbb{R}^{n}$, respectively (with constant $C_{0}$ ), then for every measure $\mu$ on $\mathbb{R}^{n}$ satisfying (i) and (ii) we have

$$
\left(C_{1} C_{2} e^{C_{0}}\right)^{-2} \mu(D)^{1 / n} \leq \mu(K)^{1 / n} \leq\left(C_{1} C_{2} e^{C_{0}}\right)^{2} \mu(D)^{1 / n}
$$

and

$$
\mu\left(D \cap D_{1}\right)^{1 / n} \leq\left(C_{1} C_{2} e^{C_{0}}\right)^{2} \mu(K \cap B)^{1 / n} .
$$

Furthermore, for every measurable set $A \subset \mathbb{R}^{n}$ we have

$$
\mu(K+A)^{1 / n} \leq C_{1} e^{C_{0}} \mu(D+A)^{1 / n} .
$$

Conditions (3) and (5) follow respectively from Theorem 2 and the definition of an $M$-ellipsoid. Condition (4) is shown by a well-known trick: first observe that for any symmetric convex bodies $L_{1}, L_{2} \subset \mathbb{R}^{n}$ and for any $x \in \mathbb{R}^{n}$ there exists $y \in L_{2}$ such that $\left(x+L_{1}\right) \cap L_{2} \subset y+2\left(L_{1} \cap L_{2}\right)$. This easily implies that one can cover $D \cap D_{1}$ by $N(D, K) N\left(D_{1}, B\right)$ translates of $4(K \cap B)$ and (4) follows from properties (i) and (ii).

Given a symmetric convex body $K \subset \mathbb{R}^{n}$, by a position of $K$ we mean any image $\tilde{K}=u(K)$, under $u \in S L_{n}$. We say that $K \subset \mathbb{R}^{n}$ is in an $M$-position with constant $C_{0}$ if a certain homothetic image of $B_{2}^{n}$ is an $M$-ellipsoid for $K$ with constant $C_{0}$. It is easy to see that in such a case, if $\lambda:=\left(|K| /\left|B_{2}^{n}\right|\right)^{1 / n}$, then $\lambda B_{2}^{n}$ is an $M$-ellipsoid for $K$ with constant $C_{0}^{\prime}$ (where $C_{0}^{\prime}$ depends on $C_{0}$ only).

Theorem 3. Let $K \subset \mathbb{R}^{n}$ be a symmetric convex body and let $D \subset \mathbb{R}^{n}$ be an $M$ ellipsoid for $K$ with constant $C_{0}$. Let $\mu$ be a measure on $\mathbb{R}^{n}$ satisfying conditions (i) and (ii). Then

$$
c^{\prime} \leq s(K, D ; \mu):=\left(\frac{\mu(K) \mu\left(K^{0}\right)}{\mu(D) \mu\left(D^{0}\right)}\right)^{1 / n} \leq C^{\prime},
$$

where $C^{\prime}$ and $c^{\prime}>0$ depend on $C_{1}, C_{2}$ and $C_{0}$ only. Moreover, if $K$ is in an $M$-position with constant $C_{0}$, then

$$
c^{\prime \prime}\left(\min \left(\frac{|K|}{\left|B_{2}^{n}\right|}, \frac{\left|B_{2}^{n}\right|}{|K|}\right)\right)^{1 / n} \leq\left(\frac{\mu(K) \mu\left(K^{0}\right)}{\left(\mu\left(B_{2}^{n}\right)\right)^{2}}\right)^{1 / n},
$$

where $c^{\prime \prime}>0$ depends on $C_{1}, C_{2}$ and $C_{0}$.

Inequality (6) corresponds to the inverse Santaló inequality of Bourgain and Milman, and to an isomorphic form of the Santaló inequality, with respect to an $M$-ellipsoid. It should be noted here that, contrary to the case of the volume, the ratio of the products $s(K, D ; \mu)$ for a more general measure $\mu$ is not necessarily affinely invariant. The moreover part corresponds to the inverse Santaló inequality (with respect to the Euclidean ball). In this case, taking a position of a body, as well as the extra factor on the left-hand side, is necessary. First, considering $K$ as an ellipsoid $D$ with two distinct sets of axes, either very short or very long, but such that $|D|=\left|B_{2}^{n}\right|$, we see that (77) might be false for such $K$. Second, the factor of the form $\min (\lambda, 1 / \lambda)$ in (7) is also necessary, in general. To see this, for any bounded measure it is sufficient to take as $K$ a very small multiple of the Euclidean ball; then the polar will have a bounded measure, but the measure of $K$ will be close to 0 .

Let us note that as an immediate consequence of (6) we get Santaló's inequality (with a universal constant) for a large class of measures. 
Corollary 4. Let $\mu$ be a measure on $\mathbb{R}^{n}$ satisfying conditions (i) and (ii), and such that there exists $C_{4}$ such that

$$
\left(\mu(D) \mu\left(D^{0}\right)\right)^{1 / n} \leq C_{4}\left(\mu\left(B_{2}^{n}\right)\right)^{2 / n},
$$

for any ellipsoid $D \subset \mathbb{R}^{n}$. Then, for an arbitrary symmetric convex body $K \subset \mathbb{R}^{n}$, we have

$$
\left(\mu(K) \mu\left(K^{0}\right)\right)^{1 / n} \leq C^{\prime}\left(\mu\left(B_{2}^{n}\right)\right)^{2 / n},
$$

where $C^{\prime}$ depends on $C_{1}, C_{2}$ and $C_{4}$ only.

As we shall see in Proposition 6 below, the class of exponential measures which we consider here satisfies (8).

Proof of Theorem 3. The upper estimate in (6) follows from

$$
\left(\frac{\mu(K) \mu\left(K^{0}\right)}{\mu(D) \mu\left(D^{0}\right)}\right)^{1 / n} \leq\left(\frac{\mu(K+D)}{\mu(K \cap D)} \frac{\mu\left(K^{0}+D^{0}\right)}{\mu\left(K^{0} \cap D^{0}\right)}\right)^{1 / n}
$$

while the lower estimate follows from

$$
\left(\frac{\mu(D) \mu\left(D^{0}\right)}{\mu(K) \mu\left(K^{0}\right)}\right)^{1 / n} \leq\left(\frac{\mu(K+D) \mu\left(K^{0}+D^{0}\right)}{\mu(K \cap D) \mu\left(K^{0} \cap D^{0}\right)}\right)^{1 / n} .
$$

For the second part of the theorem, let $D=\lambda B_{2}^{n}, \lambda>0$, be an $M$-ellipsoid for $K$ with constant $C_{0}$. Then $D^{0}=1 / \lambda B_{2}^{n}$. Moreover, $\lambda \sim\left(|K| /\left|B_{2}^{n}\right|\right)^{1 / n}$. Assume, as we obviously may, that $\lambda \leq 1$. Then by (ii) (applied to $K=\lambda B_{2}^{n}$ ) we get

$$
\left(1 / C_{2}\right) \lambda \mu\left(B_{2}^{n}\right)^{2 / n} \leq\left(\mu\left(\lambda B_{2}^{n}\right) \mu\left(1 / \lambda B_{2}^{n}\right)\right)^{1 / n},
$$

which combined with (6) gives (7).

Recall the well-known fact (cf., e.g., [G], (10) and (21)), which follows from the Prékopa-Leindler inequality, that measures $\mu$ with log-concave densities are logconcave, that is, for non-empty bounded measurable sets $K, B \subset \mathbb{R}^{n}$ and $0<t<1$, they satisfy

$$
\mu(t K+(1-t) B) \geq \mu(K)^{t} \mu(B)^{1-t} .
$$

This is, of course, a general Brunn-Minkowski inequality for $\mu$. This then suggests the natural question of whether some form of the inverse Brunn-Minkowski inequality, as proved by Milman in [M], is valid for a more general class of measures as well. An obvious necessary condition for this (formalized in condition (iii) at the beginning of this paper) is that the measures should satisfy suitable inequalities for multiples of the Euclidean ball. The next theorem shows that under this assumption, an analogue of the inverse Brunn-Minkowski inequality indeed holds for bodies in $M$-positions.

Theorem 5. Let $K, B \subset \mathbb{R}^{n}$ be symmetric convex bodies in $M$-position. Let $\mu$ be a measure satisfying (i), (ii) and (iii). Set $\lambda_{1}:=\left(|K| /\left|B_{2}^{n}\right|\right)^{1 / n}$ and $\lambda_{2}:=$ $\left(|B| /\left|B_{2}^{n}\right|\right)^{1 / n}$. If $\lambda_{1}, \lambda_{2} \in\left[a_{1}, a_{2}\right]$, then

$$
(\mu(t K+(1-t) B))^{1 / n} \leq C^{\prime}\left(t(\mu(K))^{1 / n}+(1-t)(\mu(B))^{1 / n}\right),
$$

where $C^{\prime}$ depends on $C_{1}, C_{2}, C_{3}$ and $C_{0}$. 
Proof. If $K, B$ are two bodies in $M$-positions, then using (5) twice we get

$$
\begin{aligned}
(\mu(t K+(1-t) B))^{1 / n} & \leq C_{1} e^{C_{0}^{\prime}} \mu\left(t \lambda_{1} B_{2}^{n}+(1-t) B\right)^{1 / n} \\
& \leq C_{1}^{2} e^{2 C_{0}^{\prime}} \mu\left(\left(t \lambda_{1}+(1-t) \lambda_{2}\right) B_{2}^{n}\right)^{1 / n} \\
& \leq C_{1}^{2} e^{2 C_{0}^{\prime}} C_{3}\left(t \mu\left(\lambda_{1} B_{2}^{n}\right)^{1 / n}+(1-t) \mu\left(\lambda_{2} B_{2}^{n}\right)^{1 / n}\right) \\
& \leq C_{1}^{4} C_{2}^{2} C_{3} e^{6 C_{0}^{\prime}}\left(t \mu(K)^{1 / n}+(1-t) \mu(B)^{1 / n}\right) .
\end{aligned}
$$

In the last line we used (3) and the fact that $\lambda_{1} B_{2}^{n}$ and $\lambda_{2} B_{2}^{n}$ are $M$-ellipsoids for $K$ and $B$, respectively, with constant $C_{0}^{\prime}$.

Arguments of a similar type can be used to prove some other geometric inequalities (of an isomorphic type) for arbitrary symmetric convex bodies in $\mathbb{R}^{n}$, once a measure satisfies analogous inequalities for ellipsoids. We shall illustrate this with an example related to the so-called correlation problem. For a measure $\mu$ satisfying conditions (i) and (ii) we can prove (using (4) ) that if

$$
\mu(K \cap B)^{1 / n} \geq c(\mu(K) \mu(B))^{1 / n},
$$

where $c>0$ is an absolute constant, whenever $K, B \subset \mathbb{R}^{n}$ are ellipsoids, then the same inequality holds for any two symmetric convex bodies $K, B \subset \mathbb{R}^{n}$, with constant $c^{\prime}>0$ depending on $c$. For the Gaussian measure the correlation inequality (which is (12) with $c=1$ ) was proved in [SSZ for two ellipsoids and in [H], if one body is arbitrary and the other is an ellipsoid. This implies (12) for arbitrary symmetric convex bodies with some absolute constant $c^{\prime}>0$. Also note that in [SSZ] the inequality (12) for arbitrary symmetric convex bodies with the constant $c^{\prime}=1 / \sqrt{2}$ was proved by a different method.

The main examples we consider are exponential measures defined as follows. Let $\psi: \mathbb{R}^{+} \rightarrow \mathbb{R}$ be a $C^{2}$-function such that $\psi^{\prime} \geq 0$ and $\psi^{\prime \prime} \geq 0$. Let $\mu$ be a measure on $\mathbb{R}^{n}$ defined by

$$
\mu(K)=\int_{K} \exp (-\psi(|x|)) d x,
$$

for every Borel set $K \subset \mathbb{R}^{n}$. Then we have the following.

Proposition 6. Any measure $\mu$ defined by (13) satisfies conditions (i) and (ii). If $s_{0}$ denotes the unique value such that $s_{0} \psi^{\prime}\left(s_{0}\right)=n-1$, then $\mu$ satisfies (iii) on the intervals $\left[0, s_{0}\right]$ and $\left[s_{0}, \infty\right)$. Finally, $\mu$ satisfies (8) with $C_{4}=1$.

Proof. Condition (i) with $C_{1}=1$ follows directly from the log-concavity of $\mu$ (use (10) for $K=x+B$ and $L=-x+B$; cf. also [G]).

Let $K \subset \mathbb{R}^{n}$ be a symmetric convex body and let $\|\cdot\|$ denote the norm corresponding to $K$. Then by integrating in polar coordinates we get

$$
\mu(K)=\left|S_{n-1}\right| \int_{S_{n-1}}\left(\int_{0}^{1 /\|x\|} r^{n-1} e^{-\psi(r)} d r\right) d \sigma(x),
$$

where $\sigma$ is a normalized Haar measure on $S_{n-1}$.

For $t>0$, let

$$
f(t):=\left(\int_{0}^{t} r^{n-1} e^{-\psi(r)} d r\right)^{1 / n}
$$


First note that for any $\lambda \geq 1$ and every $x \in S_{n-1}$ we have

$$
\begin{aligned}
f(\lambda /\|x\|)^{n} & =\int_{0}^{\lambda /\|x\|} r^{n-1} e^{-\psi(r)} d r=\int_{0}^{1 /\|x\|} \lambda^{n} s^{n-1} e^{-\psi(\lambda s)} d s \\
& \leq \lambda^{n} \int_{0}^{1 /\|x\|} s^{n-1} e^{-\psi(s)} d s=\lambda^{n} f(1 /\|x\|)^{n},
\end{aligned}
$$

where the inequality follows from the fact that $\psi$ is an increasing function. Integrating over $S_{n-1}$ and using (14) we immediately get (ii).

We pass now to the second part of the proposition. Let $\varphi(r):=r^{n-1} e^{-\psi(r)}$. It is interesting to note that $s_{0}$ is the point where the function $\varphi(t)$ attains its maximum.

First we shall show that $f$ is concave. We have

$$
n f^{\prime \prime}(t)=-\frac{n-1}{n}\left(\int_{0}^{t} \varphi(r) d r\right)^{\frac{1}{n}-2} \varphi(t)^{2}+\left(\int_{0}^{t} \varphi(r) d r\right)^{\frac{1}{n}-1} \varphi^{\prime}(t) .
$$

We claim that this is $\leq 0$ for all $t$. Since

$$
\varphi^{\prime}(t)=\left(\frac{n-1}{t}-\psi^{\prime}(t)\right) \varphi(t) \leq 0 \quad \text { if } \quad t \psi^{\prime}(t) \geq n-1,
$$

then $f^{\prime \prime}(t) \leq 0$ holds for all $t$ such that $t \psi^{\prime}(t) \geq n-1$. Assume next that $t \psi^{\prime}(t)<$ $n-1$. Then $\varphi^{\prime}(t) \geq 0$. Since

$$
\left(t^{n} e^{-\psi(t)}\right)^{\prime}=n t^{n-1} e^{-\psi(t)}-\psi^{\prime}(t) t^{n} e^{-\psi(t)},
$$

then we have

$$
\begin{aligned}
n \int_{0}^{t} \varphi(r) d r & =t^{n} e^{-\psi(t)}+\int_{0}^{t} r \psi^{\prime}(r) \varphi(r) d r \\
& \leq t^{n} e^{-\psi(t)}+t \psi^{\prime}(t) \int_{0}^{t} \varphi(r) d r
\end{aligned}
$$

since, by the assumption, $t \psi^{\prime}(t)$ is increasing in $t\left(\psi^{\prime \prime} \geq 0\right)$. We find

$$
n \int_{0}^{t} \varphi(r) d r \leq \frac{n}{n-t \psi^{\prime}(t)} t^{n} e^{-\psi(t)}=\frac{n}{n-t \psi^{\prime}(t)} t \varphi(t) .
$$

Moreover,

$$
\varphi^{\prime}(t) \leq \frac{n-1}{n t}\left(n-t \psi^{\prime}(t)\right) \varphi(t) .
$$

However, $f^{\prime \prime}(t) \leq 0$ if and only if $n\left(\int_{0}^{t} \varphi(r) d r\right) \varphi^{\prime}(t) \leq(n-1) \varphi(t)^{2}$. By (16) and (17),

$$
\begin{aligned}
n\left(\int_{0}^{t} \varphi(r) d r\right) \varphi^{\prime}(t) & \leq \frac{n}{n-t \psi^{\prime}(t)} t \varphi(t) \frac{n-1}{n t}\left(n-t \psi^{\prime}(t)\right) \varphi(t) \\
& =(n-1) \varphi(t)^{2}
\end{aligned}
$$

holds, i.e., $f^{\prime \prime}(t) \leq 0$ is true for all $t \geq 0$, so $f$ is concave.

We shall show that there is $c>0$ such that for all $\lambda_{1}, \lambda_{2} \in\left[0, s_{0}\right]$ and for all $\lambda_{1}, \lambda_{2} \in\left[s_{0}, \infty\right)$ one has

$$
\mu\left(\left(t \lambda_{1}+(1-t) \lambda_{2}\right) B_{2}^{n}\right)^{1 / n} \leq c\left(t \mu\left(\lambda_{1} B_{2}^{n}\right)^{1 / n}+(1-t) \mu\left(\lambda_{2} B_{2}^{n}\right)^{1 / n}\right),
$$

for all $0 \leq t \leq 1$. 
By (14) and the definition of the function $f$ above, for $\lambda>0$ we have

$$
\mu\left(\lambda B_{2}^{n}\right)^{1 / n}=\left|S_{n-1}\right| f(\lambda) .
$$

By the concavity of $f, f^{\prime}(t) \leq f^{\prime}(0)$, for $t>0$. Moreover,

$$
f^{\prime}(0)=\lim _{t \rightarrow 0} \frac{\varphi(t)}{n}\left(\int_{0}^{t} \varphi(r) d r\right)^{\frac{1}{n}-1}=\lim _{t \rightarrow 0} \frac{\varphi(t)}{n}\left(\int_{0}^{t} r^{n-1} d r\right)^{\frac{1}{n}-1}=1 / n^{1 / n} \leq 1 .
$$

We will show that for some $c>0$ depending only on $\psi, f\left(s_{0}\right) \geq c s_{0}$. Then by concavity, for $0<t<1$, we have

$$
c t s_{0} \leq t f\left(s_{0}\right) \leq f\left(t s_{0}\right) \leq f^{\prime}(0) t s_{0} \leq t s_{0} .
$$

Hence for $\lambda_{1}, \lambda_{2} \in\left[0, s_{0}\right]$ and $t \in[0,1]$,

$$
f\left(t \lambda_{1}+(1-t) \lambda_{2}\right) \leq t \lambda_{1}+(1-t) \lambda_{2} \leq c^{-1}\left(t f\left(\lambda_{1}\right)+(1-t) f\left(\lambda_{2}\right)\right)
$$

implies (18) in the interval $\left[0, s_{0}\right]$.

To estimate $f\left(s_{0}\right)$ from below we note that

$$
f\left(s_{0}\right)=\left(\int_{0}^{s_{0}} \varphi(r) d r\right)^{1 / n} \geq e^{-\psi\left(s_{0}\right) / n}\left(\int_{0}^{s_{0}} r^{n-1} d r\right)^{1 / n} \geq e^{-\psi\left(s_{0}\right) / n} s_{0} / n^{1 / n},
$$

and that - since $\psi^{\prime}$ is increasing - then

$$
\psi\left(s_{0}\right)=\psi(0)+\int_{0}^{s_{0}} \psi^{\prime}(r) d r \leq \psi(0)+s_{0} \psi^{\prime}\left(s_{0}\right) \leq \psi(0)+(n-1) .
$$

Thus $c$ may be taken as $c=\exp (-1-\psi(0)) / 2$.

Now, for $\lambda \in\left[s_{0}, \infty\right)$, the function $f$ is concave but essentially not changing much, while $f\left(s_{0}\right) \geq c s_{0}$ and $f(\infty) \leq d s_{0}$, for some constant $d$ depending only on $\psi$. From this the desired inequality is immediate if $\lambda_{1}, \lambda_{2} \in\left(s_{0}, \infty\right)$. To estimate $f(\infty)$ from above we show that $\left(\int_{s_{0}}^{\infty} \varphi(r) d r\right)^{1 / n} \leq d_{1}$, for some absolute constant $d_{1}$. Then it follows that

$$
\begin{aligned}
f(\infty) & =\left(\int_{0}^{\infty} \varphi(r) d r\right)^{1 / n}=\left(\left(\int_{0}^{s_{0}}+\int_{s_{0}}^{\infty}\right) \varphi(r) d r\right)^{1 / n} \\
& \leq\left(e^{-\psi(0)} \int_{0}^{s_{0}} r^{n-1} d r+d_{1}^{n}\right)^{1 / n} \\
& =\left(n^{-1} e^{-\psi(0)} s_{0}^{n}+d_{1}^{n}\right)^{1 / n} \leq d_{2} s_{0}+d_{1} \leq d s_{0},
\end{aligned}
$$

as required. 
To identify $d_{1}$ and complete the proof, take the $n$th power and divide by $\varphi\left(s_{0}\right)$ to get

$$
\begin{aligned}
\int_{s_{0}}^{\infty} \frac{\varphi(r)}{\varphi\left(s_{0}\right)} d r & =\int_{s_{0}}^{\infty}\left(\frac{r}{s_{0}}\right)^{n-1} \exp \left(-\left(\psi(r)-\psi\left(s_{0}\right)\right)\right) d r \\
& \leq \int_{s_{0}}^{\infty}\left(\frac{r}{s_{0}}\right)^{n-1} \exp \left(-\psi^{\prime}\left(s_{0}\right)\left(r-s_{0}\right)\right) d r \\
& =\int_{0}^{\infty}\left(\frac{s+s_{0}}{s_{0}}\right)^{n-1} \exp \left(-\psi^{\prime}\left(s_{0}\right) s\right) d s \\
& =\left(\int_{0}^{s_{0}}+\int_{s_{0}}^{\infty}\right)\left(\frac{s+s_{0}}{s_{0}}\right)^{n-1} \exp \left(-\psi^{\prime}\left(s_{0}\right) s\right) d s \\
& \leq 2^{n-1} s_{0}+2^{n-1} \int_{s_{0}}^{\infty}\left(\frac{s}{s_{0}}\right)^{n-1} \exp \left(-\psi^{\prime}\left(s_{0}\right) s\right) d s
\end{aligned}
$$

Substituting $u=\psi^{\prime}\left(s_{0}\right) s$ and using that $s_{0} \psi^{\prime}\left(s_{0}\right)=n-1$, the latter expression is equal to

$$
\begin{aligned}
& 2^{n-1} s_{0}+2^{n-1} / \psi^{\prime}\left(s_{0}\right) \int_{s_{0}}^{\infty}\left(\frac{u}{n-1}\right)^{n-1} e^{-u} d u \\
& \leq 2^{n-1} s_{0}+2^{n-1} / \psi^{\prime}\left(s_{0}\right)(n-1) ! /(n-1)^{n-1} .
\end{aligned}
$$

(We estimated the integral in the last step by the integral from 0 to $\infty$.)

Therefore

$$
\left(\int_{s_{0}}^{\infty} \varphi(r) d r\right)^{1 / n} \leq 2\left(s_{0}+1 / \psi^{\prime}\left(s_{0}\right)\right)^{1 / n} \varphi\left(s_{0}\right)^{1 / n} \leq d_{1}
$$

for some absolute constant $d_{1}$, since $s_{0}=O(n)$. This completes the proof of the case of the interval $\left[s_{0}, \infty\right)$.

The last statement follows from the recent result by Cordero, Fradelizi and Maurey [CFM] where, among other results, connections between complex interpolation and the Brunn-Minkowski and Santaló inequalities have been developed. For the reader's convenience we briefly describe it here. They call a function $\varphi: \mathbb{R}^{n} \rightarrow \mathbb{R}$ unconditional if $\varphi\left(x_{1}, \ldots, x_{n}\right)=\varphi\left(\left|x_{1}\right|, \ldots,\left|x_{n}\right|\right)$, for $\left(x_{1}, \ldots, x_{n}\right) \in \mathbb{R}^{n}$. A set $K \subset \mathbb{R}^{n}$ is unconditional if its characteristic function is unconditional (in other words, the standard unit vector basis is a 1 -unconditional basis in $\left(\mathbb{R}^{n}, K\right)$ ). In Proposition 7 of [CFM] they consider an unconditional function $\varphi: \mathbb{R}^{n} \rightarrow \mathbb{R}$ such that $\left(r_{1}, \ldots, r_{n}\right) \rightarrow \varphi\left(e^{r_{1}}, \ldots, e^{r_{n}}\right)$ is convex and the measure $\mu$ on $\mathbb{R}^{n}$ is defined by $\mu(K)=\int_{K} \exp (-\varphi(x)) d x$ for every Borel set $K \subset \mathbb{R}^{n}$. Then they prove that for any two unconditional bodies $L_{1}, L_{2} \subset \mathbb{R}^{n}$ and every $\theta \in[0,1]$ one has

$$
\mu\left(L_{1}^{1-\theta} L_{2}^{\theta}\right) \geq \mu(K)^{1-\theta} \mu(L)^{\theta} .
$$

(Here we use the notation

$$
L_{1}^{1-\theta} L_{2}^{\theta}:=\left\{\left.w \in \mathbb{R}^{n}\left|\exists x \in L_{1}, y \in L_{2},\right| w_{j}|=| x_{j}\right|^{1-\theta}\left|y_{j}\right|^{\theta}, \text { for } j=1, \ldots, n\right\}
$$

analogous to the familiar Calderon's complex interpolation formula for function spaces.)

Now notice that a measure $\mu$ defined by (13) satisfies the above hypothesis and is rotation invariant. Let $D \subset \mathbb{R}^{n}$ be an ellipsoid; and without loss of generality assume, as we may, that its semi-axes are in the directions of the standard 
unit vector basis, that is, $D$ is unconditional. Finally recall a familiar fact that $D^{1 / 2}\left(D^{0}\right)^{1 / 2}=B_{2}^{n}$ (recently used in [C] in the complex case). Using this fact and (19) the same way as in [C], Corollary 3.3 , we get (8) with $C_{4}=1$.

At the end of this note let us consider the following well-known example: for a fixed symmetric convex body $L \subset \mathbb{R}^{n}$, let

$$
\mu_{L}(K)=\frac{|K \cap L|}{|L|}
$$

for every Borel set $K \subset \mathbb{R}^{n}$.

Then every measure $\mu_{L}$ is log-concave, and hence it satisfies condition (i). Condition (ii) is trivial, however (iii) is not necessarily satisfied. Namely, given an interval $\left[a_{1}, a_{2}\right]$ with $a_{1}<a_{2}$ we may always find $L$ (namely $L=\varepsilon B_{2}^{n}$ for an appropriately chosen $\varepsilon>0$ ) such that $\mu_{L}$ does not satisfy (iii) on $\left[a_{1}, a_{2}\right]$.

\section{ACKNOWLEDGMENT}

We would like to thank Bernard Maurey for several stimulating discussions.

\section{REFERENCES}

[BM] J. Bourgain And V. D. Milman, New volume ratio properties for symmetric bodies in $\mathbb{R}^{n}$. Invent. Math. 88 (1987), no 2, 319-340. MR0880954 (88f:52013)

[C] D. Cordero-Erausquin, Santaló's inequality on $\mathbb{C}^{n}$ by complex interpolation. C. R. Acad. Sci. Paris Sér. I Math. 334 (2002), 767-772. MR1905037 (2003f:32042)

[CFM] D. Cordero-Erausquin, M. Fradelizi and B. Maurey, The (B) conjecture for the Gaussian measure of dilates of symmetric convex sets and related problems. preprint.

[G] R. J. Gardner, The Brunn-Minkowski inequality. Bull. Amer. Math. Soc. 39 (2002), 355-405. MR1898210 (2003f:26035)

[H] G. Hargé, Une inégalité de décorrélation pour la measure Gaussienne. C. R. Acad. Sci. Paris Sér. I Math. 326 (1998), 1325-1328. MR1649146|(99h:60081)

[M] V. D. Milman, An inverse form of the Brunn-Minkowski inequality, with applications to the local theory of normed spaces. C. R. Acad. Sci. Paris Sér. I Math. 302 (1986), 25-28. MR0827101 (87f:52018)

[P] G. PISIER, Volumes of Convex Bodies and Banach Space Geometry. Cambridge Univ. Press, 1989. MR1036275 (91d:52005)

[SSZ] G. Schechtman, Th. Schlumprecht and J. Zinn, On the Gaussian measure of the intersection. Ann. Probab. 26 (1998), 346-357. MR1617052 (99c:60032)

Mathematisches Seminar, Universitaet Kiel, Ludewig-Meyn-Strasse 4, D-24098 Kiel, Germany

E-mail address: hkoenig@math.uni-kiel.de

Department of Mathematical and Statistical Sciences, University of Alberta, Edmonton, Alberta, Canada T6G 2G1

E-mail address: nicole@ellpspace.math.ualberta.ca 